\title{
NOURSHING HABITS OF PEOPLE WITH DISABILITIES
}

\author{
Romanova Hr., R. Radev ${ }^{1}$ \\ Medical University - Varna, ${ }^{1}$ Society of disabled - Varna
}

Reviewed by: assoc. prof. B. Yustiniyanova

\section{RESUME}

Research is leaded for the nourishing habits of 130 people with disabilities from the Society of disabled Varna. The nourishing habits of the inquired are unhealthy and with unfavourable consequences, leading to obesity and change for the worse of the general state of health. The nutrition of this group manifests big (daily) consummation of sugar, sugar nourishment and sweets from almost half of the inquired, which raised the obesity. The consummation of fish, fresh fruits and vegetables, milk and dairy products is low because of the unsufficient financial means for providing of the health nutrition. The predominant part of the included people in the research (almost 2/3) do not add salt in addition in the food or consummate salt-free and do not use alcohol in general or rarely. Only half of the inquired try to change the nourishing habits with the purpose of reducing the weight and preserving health.

Key words: nourishing habits, people with disabilities

\section{INTRODUCTION}

Wrong eating habits are the main reason, which alone or in combination with medical prblem (physical or mental impairment) significantly restrict the invalid's opportunity to have a complete way of life (3).

In raised by us in 2005 research on the model of eating behavior of a group of disabled from Varna it found a high incidence of obesity (4). Obesity is a global epidemic in developed countries and contributes to further damage the state, especially people with permanently impaired health. Centers for Disease Control in the U.S. (CDC) reported that $30 \%$ of adults - over 20 years (about 60 million people) are overweight (6). According to WHO 36\% of Europe's population is overweight or obesed. In Bulgaria $35 \%$ of women and $25 \%$ of men are obesed (10).

Another study in the U.S. suggests that people with disabilities have a very high percentage of obesity: in white - $54 \%$, African Americans - 70\% Hispanic - 44\% (7).

Our aim was to study the eating habits of people with permanently impaired health and to determine body mass index (BMI) of study participants.

\section{MATERIALS AND METHODS}

Conducted anonymous survey with an individual questionnaire and a standardized interview of 130 people, members of

\footnotetext{
Address for correspondence:

Hr. Romanova, Disaster situations medicine,

Medical University Prof. Dr. Paraskev Stoyanov - Varna

e-mail: romanova_hr@abv.bg
}

the Society of the Disabled - Varna, by random selection of participants. There are 30 questions - 25 pre-formulated answer 4 questions were open and given you the opportunity for another opinion and a question in the form of a table in which respondents indicate the frequency of use (1 time per day, more than once daily, 1 time weekly, etc.) of the listed foods.

Respected the principle of equity to different personalities, ages, human qualities, occupation or degree of permanent impaired health.

To assess the nutritional anthropometric status was used Body mass index (BMI) of participants.

It was created a computer processing of results.

\section{RESULTS AND DISCUSSION}

Enrolled people with permanently impaired health have received their disability as a result primarily of: about $60 \%$ with cardiovascular disease, 1/6 - cerebrovascular disease, 1/10 - complications of diabetes, $1 / 10$ - complications malignant tumors with different location, injuries and other diseases.

The research of eating habits shows that majority of respondents have a three-feeding $\operatorname{diet}(66.17 \%)$ with double-feeding were $19.23 \%$, four-feeding $-11.75 \%$ and a very small proportion of single meal $-3,85 \%$.

Systemic daily consumation of bread and pasta from 93.08 percent of the respondents scored as correct and meets the global dietary guidelines. WHO recommends cereals to be based on healthy dietary pattern. (10)

Milk and milk products have many others valuable ingredients - high quality protein, easily absorbed calcium, almost all vitamins and nutritionists recommend them for every- 
summation. But from the studied group of people with permanently impaired health only one third accept these foods daily, 41.54 percent consummate them 1 to 3 times a week and $1 / 4$ 1 to 3 times a month, which is extremely insufficient.

Regular consummation of meat - daily or 1 to 3 times a week reported 68.46 percent of the respondents. To decrease the risk of cardiovascular disease WHO recommended intake mainly chicken and fish (9). Consummation of chicken is good, but consummation of fish is inadequate. Only 1/4 adopt fish 1 to 3 times a week and $53.54 \%$ consumed fish 1-3 times a month. Our research about the insufficiently fish consummation coincide with the data from the National Nutrition Survey of popiulation in Bulgaria in 1998 (2).

Taking the eggs is sufficient for $45 \%$ of respondents.

Pulses are a traditional food for Bulgarians. They are a good source of protein, complex carbohydrates and fiber. Nearly half of survey respondents include legumes in their diet - $44.61 \%$ weekly, $33.84 \%$ - 1 to 3 times a month and the remaining $21.55 \%$ rarely consume almost no pulse, which is explained by medical reasons.

A significant percentage of those taking daily sugar confectionery $(46.15 \%)$, which we appreciate as unhealthy. This leads to an increase in energy imports, hyperglycemia and increased risk of obesity and diabetes. We explain this fact with the wide variety and relatively low prices and delicious type of goods offered.

Fresh fruit and vegetables are an indispensable source of vitamins and minerals, and WHO recommends their daily consumption. Only $1 / 4$ of the respondents comply with that recommendation, but about $1 / 2$ take them 1 to 3 times a week. Fresh fruits and vegetables consummation is insufficient. Perhaps the matter and the period during which the study took place (winter - spring). Prices are high and are not accessible to low income people with impaired health.

Sodas are not used frequently by people with disabilities, $90 \%$ consuming them rarely less than 1-3 times a month. Daily coffee drinking was observed only in $41.54 \%$ of respondents.

The majority of enrolled (63.85\%) did not add extra salt to food or eat unsalted, which is beneficial to their health. It is well known that high consummation of salt is a risk factor for cardiovascular diseases.

$2 / 3$ of respondents do not use never or rarely drink alcohol, which established and other authors among the economic nactive population (1).

The question "Which diseases are related to diet?" 90\% indicate obesity, $76 \%$ - high blood pressure, $68.66 \%$ - osteoporosis, $66.16 \%$ - atherosclerosis, $35.38 \%$ - risk diseases. Responses in the survey show that respondents have knowledge about the impact of food on human health, but socio-economic status has an adverse impact on their eating habits.

Anthropometric data to assess the nutritional status show that a normal body mass (BMI) were only 24.61 percent of respondents overweight $-26.93 \%$ and in varying degrees of obesity - one half (1/2).

Overweight and obesity are a result of reduced physical activity of people with permanently impaired health as a consequence of disability. But the strengthening of these ad- verse indicators influence and dietary habits. Reduced physical activity leads to reduction of muscle mass and increase fat. Obesity leads to deterioration of cardiovascular, endocrine, cancer and other diseases and ultimately to a worsening of overall health.

Only $1 / 3$ (round 31.54 percent ) recognize that make a diet in relation to their disease. When asked in detail about changing dietary habits reported only half of the respondents: $19.23 \%$ - to lose weight, $13.85 \%$ - not to gain weight and 16.92 percent - to maintain their health.

\section{CONCLUSIONS}

1. Food habits of the surveyed people with permanently impaired health are unhealthy and adverse effects leading to obesity and deterioration of general health.

2. Nutrition research group showed high (daily) consumation of sugar confectionery and food for nearly half of respondents, increasing obesity.

3. Lower the consummation of fish, fresh fruits and vegetables, milk and milk products due to insufficient financial resources to ensure healthy eating.

4. The majority of enrolled (about 2/3) do not add extra salt to food or feed use unsalted and never or rarely drink alcohol, which is beneficial to their health.

5. Only half of the respondents are trying to change their eating habits in order to - reduce weight and maintain health.

\section{LITERATURE}

1. Ovcharova E. Socio-economic status and diet, $H y$ giene and health, 2000, 62-67

2. Pertova S. Food consummation and co-author. National survey of dietary and nutritional status of the population in Bulgaria, 1998, Hygiene and Public Health, 2000, 3-4, 55-62

3. Popova E. O. Vasilkovska N. Hadjipetrov, S. Velkova Vitkina M., Study on treatment of patients with collateral cardiovascular disease, nutrition science to new opportunities and challenges, Sofia, 2008

4. Romanova Hr. K. Petrova, N. Radeva, Nutritional status and food behavior of disabled people from Varna, Social Medicine, 4, 2006

5. Bjontorp P. Coronary disease and obesity, Medicographia, 13, 2, 1991, 45-47

6. Baldini, L. Obesity among growing up subjects of limited mobility. Boston, Massachusetts, 2005 (in Bulgarian).

7. Nosek, P. Over-weight and obesity among physically damaged females. Waco, TX, Baylor University, 2008 (in Bulgarian).

8. Rimmer, D., M. Kembel. Obesity differences among damaged people. Austin, TX, SEDL, National Center for the Dissemination of Disability Research, Webcast, 3.12.2009 (in Bulgarian).

9. Rosenbaum M, Leibel R, Hirsch J., Obesity, N. Engli J. Med 337,6,1997, 396-407

10. WHO (2000) CINDI dietary guide. 